\title{
ON THE LOCALIZATION AND DIRECTIONALIZATION OF UNIFORM CONVEXITY ${ }^{1}$
}

\author{
BY DENNIS F. CUDIA ${ }^{2}$ \\ Communicated by M. M. Day, October 22, 1962
}

1. Introduction. There are two main theorems in this note. The first states the close connection between the localization and directionalization of uniform convexity in a normed space $X$ and various differentiability conditions satisfied by the norm in $X^{*}$. This answers a question implicit in $[1 ; 5]$ and makes possible the extension of the dual theory of differentiability of the norm as initiated in $[6 ; 7]$. The second main theorem examines the spherical image map defined on certain infinite dimensional manifolds imbedded in $X$ and gives characterizations of the smoothness of the manifold in terms of the continuity properties of the spherical image map in various topologies in the spaces $X$ and $X^{*}$. These results can be used to solve a problem proposed in [4].

Notation. $X$ will be an arbitrary normed linear space with the reals as scalar field. $U=\{x:\|x\| \leqq 1, x \in X\}, S=\{x:\|x\|=1, x \in X\}$ and $U^{\prime}$ and $S^{\prime}$ denote the analogous sets in $X^{*} . Q$ is the canonical map imbedding $X$ into $X^{* *}$. When it is to be emphasized that the conjugate norm in $X^{*}$ is being considered, this norm will be denoted by $\|\cdot\|^{*} \cdot \theta$ is the neutral element of a linear space.

2. Modifications of uniform convexity and differentiability of the norm. Define the modulus of uniform convexity by

$$
\delta(\epsilon)=\inf _{\|x-v\| \geqq \epsilon ; x, v \in S} 2-\|x+y\| .
$$

In the next few definitions let $f$ and $g$ be fixed elements in $S^{\prime}$. Then the modulus of weak uniform convexity at $f$ is defined by

$$
\delta(\epsilon, f)=\inf _{\|x-y\| \geq \epsilon ; x, y \in S} 2-|f(x+y)| ;
$$

the modulus of uniform convexity in the direction $g$ is defined by

$$
\delta(\epsilon, g)=\inf _{|\theta(x-y)| \geqq \epsilon ; x, y \in S} 2-\|x+y\| ;
$$

1 This note is a summary of a part of a dissertation and submitted in partial fulfillment of the requirements for the Ph.D. degree at the University of Illinois (Urbana).

2 Author supported by contracts NSF G 12171 and NSF G 19869. 
and the modulus of weak uniform convexity at $f$ in the direction $g$ is defined by

$$
\delta(\epsilon, f, g)=\inf _{|\rho(x-y)| \geq \epsilon ; x, y \in S} 2-|f(x+y)| .
$$

$X$ will be said to be uniformly convex (see [2]), weakly uniformly convex at $f$, uniformly convex in the direction $g$, or weakly uniformly convex at $f$ in the direction $g$ provided the corresponding modulus is positive when $0<\epsilon<2$.

Let $x, y \in S$ and consider the limit

$$
G^{+}(x, y)=\lim _{\alpha \rightarrow 0^{+}} \frac{\|x+\alpha v\|-\|x\|}{\alpha} .
$$

Let $G^{-}(x, y)=-G^{+}(x,-y)$. Then $\|\cdot\|$ is said to be differentiable at $x$ with respect to $y$ provided $G^{+}(x, y)=G^{-}(x, y) ;\|\cdot\|$ is said to be Fréchet differentiable at $x$ provided $G^{+}(x, y)=G^{-}(x, y)$ for all $y$ in $S$ and the limit $\left(^{*}\right)$ is approached uniformly as $y$ varies over $S ;\|\cdot\|$ is said to be uniformly Gateaux differentiable in the direction $y$ provided $G^{+}(x, y)=G^{-}(x, y)$ for all $x$ in $S$ and the limit (*) is approached uniformly as $x$ varies over $S ;\|\cdot\|$ is said to be uniformly Fréchet differentiable provided $G^{+}(x, y)=G^{-}(x, y)$ for all $x$ and $y$ in $S$ and the limit $\left(^{*}\right)$ is approached uniformly as $x$ and $y$ vary over $S$.

In the next theorem $X$ is an arbitrary normed linear space over the reals and $\|x\|=\|y\|=\|f\|^{*}=\|g\|^{*}=1$. Dual results are indicated by primes. Smulian [7] gives (iv) and (iv') and the sequentialized forms of (ii) and (ii').

THEOREM 1. (i) $\|\cdot\|^{*}$ is differentiable at $f$ with respect to $g$ iff $X$ is weakly uniformly convex at $f$ in the direction $g$. (ii) $\|\cdot\|^{*}$ is Fréchet differentiable at $f$ iff $X$ is weakly uniformly convex at $f$. (iii) $\|\cdot\| *$ is uniformly Gateaux differentiable in the direction $g$ iff $X$ is uniformly convex in the direction $g$. (iv) $\|\cdot\| *$ is uniformly Fréchet differentiable iff $X$ is uniformly convex. $\left(\mathrm{i}^{\prime}\right)\|\cdot\|$ is differentiable at $x$ with respect to $y$ iff $X^{*}$ is weakly uniformly convex at $Q x$ in the direction $Q y$. (ii') $\|\cdot\|$ is Fréchet differentiable at $x$ iff $X^{*}$ is weakly uniformly convex at $Q x$. (iii') $\|\cdot\|$ is uniformly Gateaux differentiable in the direction $y$ iff $X^{*}$ is uniformly convex in the direction $Q y$. (iv') $\|\cdot\|$ is uniformly Fréchet differentiable iff $X^{*}$ is uniformly convex.

3. The spherical image map. Let $K$ be a closed bounded convex set with an interior point in the norm topology of $X$. For each point $x$ on the boundary of $K$ let $\nu_{x}$, the spherical image of $x$, be the set of linear functionals of norm one with the property that the inverse image of a positive number is a hyperplane of support of $K$ at $x$. 
Denote by $\|\cdot\|_{1}$ a norm equivalent to $\|\cdot\|$ and let $U_{1}=\left\{x:\|x\|_{1} \leqq 1\right.$, $x \in X\}, S_{1}=\left\{x:\|x\|_{1}=1, x \in X\right\}$. In the next theorem the infinite dimensional manifolds $S$ and $S_{1}$ are considered and in the latter case the spherical image map $\nu$ is defined as above by setting $U_{1}=K$. The "only if" part of (i) is given in [6].

THEOREM 2. (i) $\|\cdot\|$ is differentiable at any $x$ on $S$ with respect to any $y$ on $S$ iff the map $\nu$ defined on $S$ is lower semicontinuous from the norm topology on $S$ into the weak* topology on $S^{\prime}$. (ii) $\|\cdot\|$ is uniformly Gateaux differentiable in any direction $y$ on $S$ iff the map $\nu$ defined on $S$ is single valued and uniformly continuous from the norm topology on $S$ into the weak* topology on $S^{\prime}$. (iii) Let $\nu$ be defined on $S_{1}$. Then $\|\cdot\|_{1}$ is [uniformly] Fréchet differentiable iff $\nu$ is single valued and [uniformly] continuous from the norm topology on $S_{1}$ into the norm topology on $S^{\prime}$.

4. Application. Define the extended spherical image map $T$ from $X$ into $X^{*}$ by setting $T \theta=\theta$ and $T x=\|x\| \nu_{\|x\|^{-1} x}$ for $x \neq \theta$. It is well known that if $X$ is a real Hilbert space (so that $X$ is uniformly convex and uniformly Fréchet differentiable) then $T$ is a linear isometry between $X$ and $X^{*}$. Klee [4, p. 35] has posed the following problem: Characterize intrinsically those spaces $X$ for which $T$ is a homeomorphism of $X$ onto $X^{*}$ in the norm topologies.

The main theorems of this note can be combined to give the following answer to this problem.

TheOREM 3. $T$ is a homeomorphism iff $X$ is a weakly uniformly convex Banach space with a Fréchet differentiable norm.

The proofs of these and other theorems with other applications are to be found in [3].

\section{REFERENCES}

1. K. W. Anderson, Midpoint local uniform convexity, and other geometric properties of Banach spaces, Doctoral dissertation, Univ. of Illinois, Urbana, Ill., 1960.

2. J. A. Clarkson, Uniformly convex spaces, Trans. Amer. Math. Soc. 40 (1936), 396-414.

3. D. F. Cudia, The geometry of Banach spaces. Smoothness, Trans. Amer. Math. Soc. (to appear).

4. V. L. Klee, Convex bodies and periodic homeomorphisms in Hilbert space, Trans. Amer. Math. Soc. 74 (1953), 10-43.

5. A. R. Lovaglia, Locally uniformly convex Banach spaces, Trans. Amer. Math. Soc. 78 (1955), 225-238.

6. V. L. Smulian, On some geometrical properties of the unit sphere in the space of the type (B), Mat. Sb. (N.S.) 48 (6) (1939), 77-94. (Russian. English summary, 90-94).

7. - Sur la dérivabilité de la norme dans l'espace de Banach, Dokl. Akad. Nauk SSSR 27 (1940), 643-648.

UNIVERSITY OF ILLINOIS 